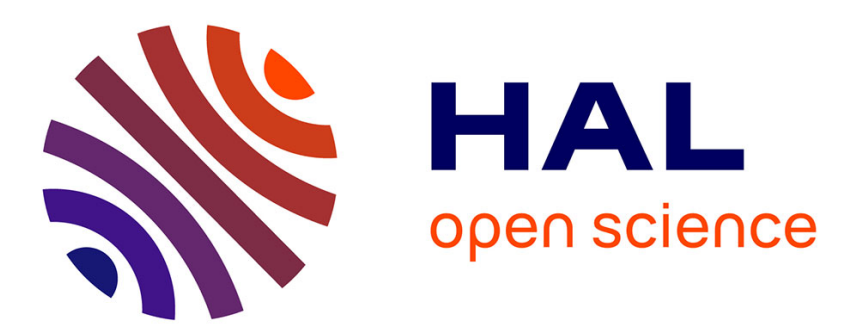

\title{
Bi-stability of a pendular disk in laminar and turbulent flows
}

\author{
Martin Obligado, Martin Puy, Mickaël Bourgoin
}

\section{To cite this version:}

Martin Obligado, Martin Puy, Mickaël Bourgoin. Bi-stability of a pendular disk in laminar and turbulent flows. Journal of Fluid Mechanics, 2013, 728, pp.R2. 10.1017/jfm.2013.312 . hal-01924959

\section{HAL Id: hal-01924959 \\ https://hal.science/hal-01924959}

Submitted on 5 Jun 2020

HAL is a multi-disciplinary open access archive for the deposit and dissemination of scientific research documents, whether they are published or not. The documents may come from teaching and research institutions in France or abroad, or from public or private research centers.
L'archive ouverte pluridisciplinaire HAL, est destinée au dépôt et à la diffusion de documents scientifiques de niveau recherche, publiés ou non, émanant des établissements d'enseignement et de recherche français ou étrangers, des laboratoires publics ou privés. 


\title{
Bi-stability of a pendular disk in laminar and turbulent flows
}

\author{
M. Obligado ${ }^{1} \dagger$, M. Puy ${ }^{1}$ \\ and M. Bourgoin ${ }^{1}$ \\ ${ }^{1}$ Laboratoire des Écoulements Géophysiques et Industriels, CNRS/UJF/G-INP UMR 5519, \\ Université de Grenoble, BP53, 38041, Grenoble, France
}

(Received )

The simple pendulum remains one of the most fundamental systems studied in physics. It is commonly used as a model to illustrate a broad variety of mechanisms in a wide range of areas. However, in spite of this popularity, subtle behaviors still remain to be discovered and to be explored when the pendulum is strongly coupled to fluid mechanics. This is for instance illustrated in recent studies by Neill et al. (2007) and Bolster et al. (2010) which highlight the impact on a simple spherical pendulum of vortex shedding and added mass effects. In the present work we show that the equilibrium of a pendular disk facing a flow exhibits bi-stability and hysteresis. We give a simple interpretation of this behavior in terms of a two potential wells description, only requiring to know the angular dependency of the normal drag coefficient of an inclined plate. We investigate the influence of turbulence on the equilibrium of the pendulum in general and on the observed bi-stability in particular. Our results have potentially important fundamental and practical consequences: (i) they extend the attractiveness of the pendulum as a model to investigate generic questions related to bi-stable stochastic processes, (ii) they highlight important fluid dynamic mechanisms, including turbulent drag enhancement and fluid-structure interaction issues.

\section{Introduction}

The pendulum is a paradigm for many problems in physics (oscillators, resonances, instabilities, dissipative systems, etc.) which has been investigated for centuries and which remains one the most fundamental systems taught in academy. However, in spite of this popularity, the richness of this simple system is so that the topic is far from been exhausted. This is particularly the case when the coupling of the pendulum with its surrounding fluid is considered. In a recent study, Neill et al. (2007) have for instance investigated the role of added mass on a simple spherical pendulum, which offers an elegant framework to revisit the equivalence principle of inertial and gravitational masses. More recently, Bolster et al. (2010) have evidenced additional discrete dissipative mechanisms arising on a spherical pendulum with large amplitude oscillations, due to vortex ring shedding.

These examples illustrate that non-trivial processes emerge when the pendulum is strongly coupled to fluid mechanics. In the present work we investigate further effects which appear when a pendulum faces a mean stream instead of being surrounded by still fluid. The mean flow results in additional aerodynamic forces whose first effect is to modify the equilibrium position of the pendulum as it is dragged and/or lifted by the flow. We focus here on the characterization of the equilibrium of a simple pendulum made

$\dagger$ Email address for correspondence: mickael.bourgoin@legi.cnrs.fr 


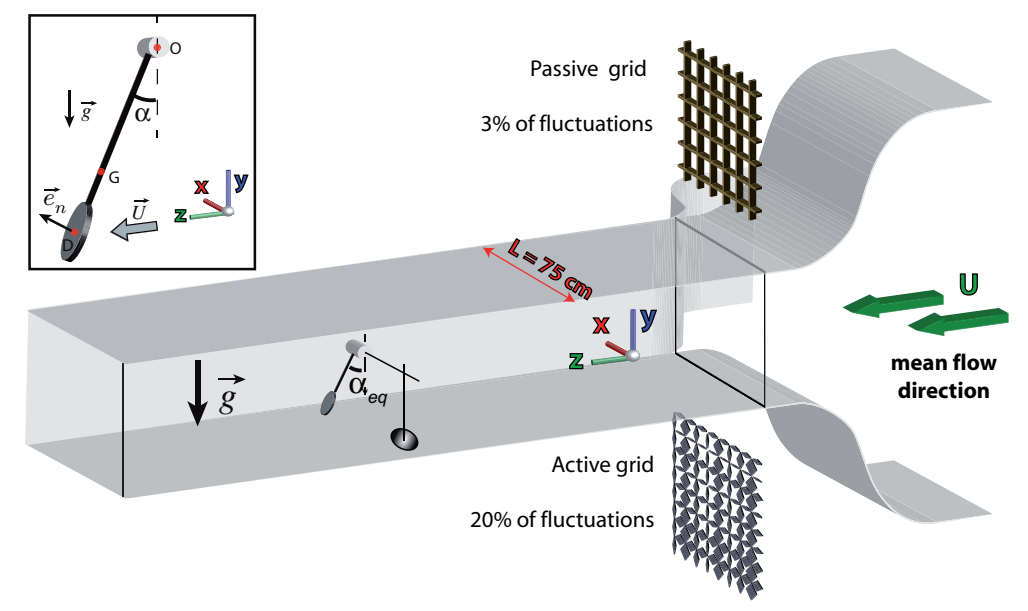

Figure 1. The experiment runs in a low turbulence wind tunnel with a $0.75 \mathrm{~m} \times 0.75 \mathrm{~m} \times 4$ test section. Explored velocities range between $1 \mathrm{~m} / \mathrm{s}$ and $20 \mathrm{~m} / \mathrm{s}$ with a turbulent velocity fluctuation rate $\phi<0.5 \%$. A grid can be inserted at the entrance of the test section to increase the level of turbulence. The pendulum is located far downstream the entrance of the test section where the turbulence generated was verified to be well developed with good homogeneity and isotropy properties. The top left inset details the pendulum. It is made of a thin aluminum circular plate ( $4 \mathrm{~cm}$ in diameter, $1 \mathrm{~mm}$ in thickness) connected to the pivot axis $O x$ by a thin beveled rigid blade with negligible frontal area facing the flow. $G$ denotes the center of mass of the system disk + blade and $D$ the center of the disk.

of a circular flat plate facing a mean stream (see figure 1). Already in 1450, the italian mathematician Leon Battista Alberti imagined to use such a system as an anemometer, the inclination of the swing plate giving the wind velocity. Leonardo da Vinci and Robert Hooke recovered Battista's idea and built such a swing plate anemometer in 1500 and 1667 respectively. However, in spite of these early developments, although the case of fixed inclined disks and free falling plates, have received significant attention (see for instance the recent numerical studies by Auguste et al. (2013) and by Chrust et al. (2013) on instabilities of thin free falling disks), the study of the pendular plate configuration seems to have remained overlooked. We show in the present work that despite its apparent simplicity, this system reveals an unexpected richness including: (i) bi-stability and hysteretical behavior of the pendulum as the flow velocity is cycled up and down, (ii) influence of incident turbulence on the bi-stability and (iii) evidence of drag enhancement in highly turbulent conditions. Implications of these results are important, both from a fundamental point of view (in the context for instance of fluid-structure interactions and stochastic processes in bi-stable systems) and for practical fluid-structure interaction applications, as such swing plate systems are common elements in artificial heart valves and nuclear plant check valves among others.

\section{Experimental setup}

Our experiment runs in a low-turbulence wind-tunnel (see figure 1). A turbulence generating grid can be introduced at the entrance of the test section. Three configurations of flow were investigated : (i) laminar, with no grid at the entrance, the velocity fluctuation rate, $\phi=\sigma_{u} / U$ (where $\sigma_{u}$ is the standard deviation of velocity fluctuations and $U$ is the average stream velocity) remains then below $0.5 \%$; (ii) moderately turbulent ( $\phi \simeq 3 \%$ ) produced with a passive grid and (iii) highly turbulent $(\phi \simeq 20 \%$ ), with an active grid (see Obligado et al. (2011) for a detailed description). For each configuration, we monitor 
$[\mathrm{b}]$

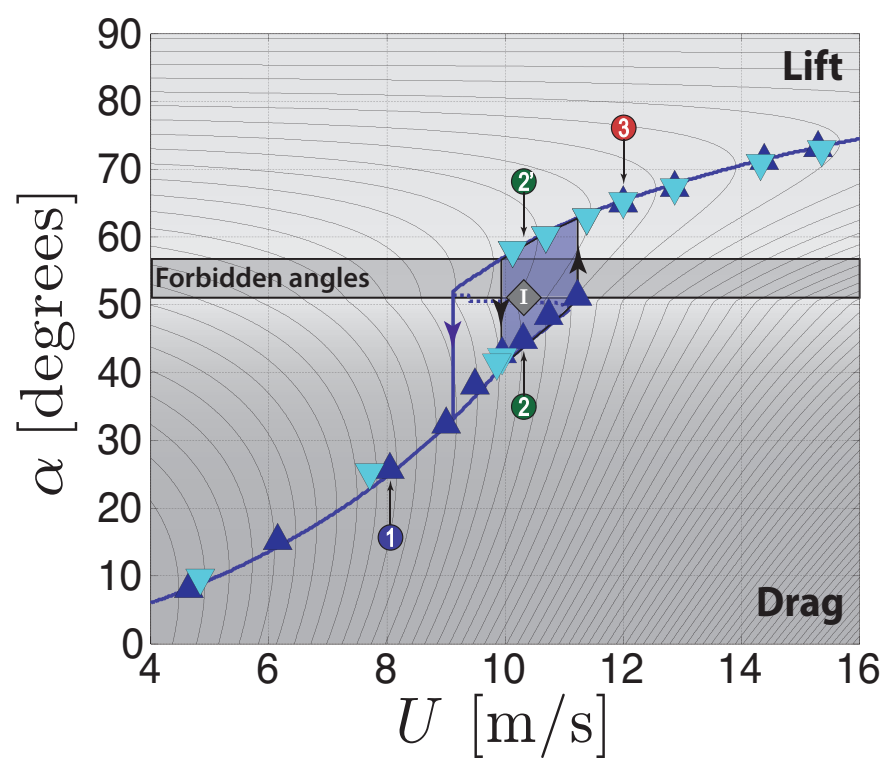

FIGURE 2. Equilibrium angle measured experimentally for increasing (dark blue $\triangle$ ) and decreasing (light blue $\nabla$ ) wind velocity. Lines in the background represent iso-contours of the total potential energy of the system (gravity + aerodynamic) as further illustrated in figure 3d. The blue solid line indicates the prediction of the stable equilibrium angle from the path of local minima of total potential energy. Labeled points corresponds to the equilibrium analysis discussed in figure 3 . Dark gray bottom region indicates low inclination states (namely $\alpha<\alpha^{\text {stall }}$ ) where aerodynamic force is dominated by drag, while light gray upper region indicates high inclination states, where lift dominates.

the equilibrium angle $\alpha_{e q}$ of the pendulum (using a high precision rotating angular sensor at the pendulum pivot) as a function of the mean wind velocity $U$. The pendulum is made of a thin aluminum disk ( $4 \mathrm{~cm}$ in diameter, $1 \mathrm{~mm}$ in thickness). The length $\overline{O D}$ between the pivot of the pendulum $O$ and the center of the disk $D$ is $\overline{O D}=26 \mathrm{~cm}$. The disk is connected to the pivot by a thin beveled rigid blade with negligible frontal area facing the flow. The center of mass $G$ of the system blade+disk is such that $\overline{O G}=15.5 \mathrm{~cm}$. Finally we note that in the turbulent cases, the size of the disk remains smaller, but is of the order, of the energy injection scale of the flow $L_{\text {int }}\left(L_{\text {int }} \simeq 7 \mathrm{~cm}\right.$ with the passive grid, and $L_{\text {int }} \simeq 15 \mathrm{~cm}$ with the active grid).

\section{Results and discussion}

\subsection{Laminar case}

Figure 2 shows the equilibrium angle of the pendulum in the laminar case (no grid in the wind tunnel) as a function of flow velocity, when the wind speed is cycled up and down. When the wind velocity is increased from rest, the equilibrium angle of the pendulum naturally increases as the plate is dragged by the flow (dark blue triangles pointing up, $\triangle$ ). An abrupt transition from a low equilibrium branch to an upper equilibrium branch is observed for a critical velocity $U_{\uparrow}^{\text {lam }} \simeq 11.2 \mathrm{~m} / \mathrm{s}$, where $\alpha_{e q}$ suddenly jumps from around $50^{\circ}$ to around $65^{\circ}$. If we now start from a high speed configuration and the wind velocity is decreased starting from the upper branch (light blue triangles pointing down, $\nabla)$, a hysteretical behavior is observed: the reverse transition back towards the lower 

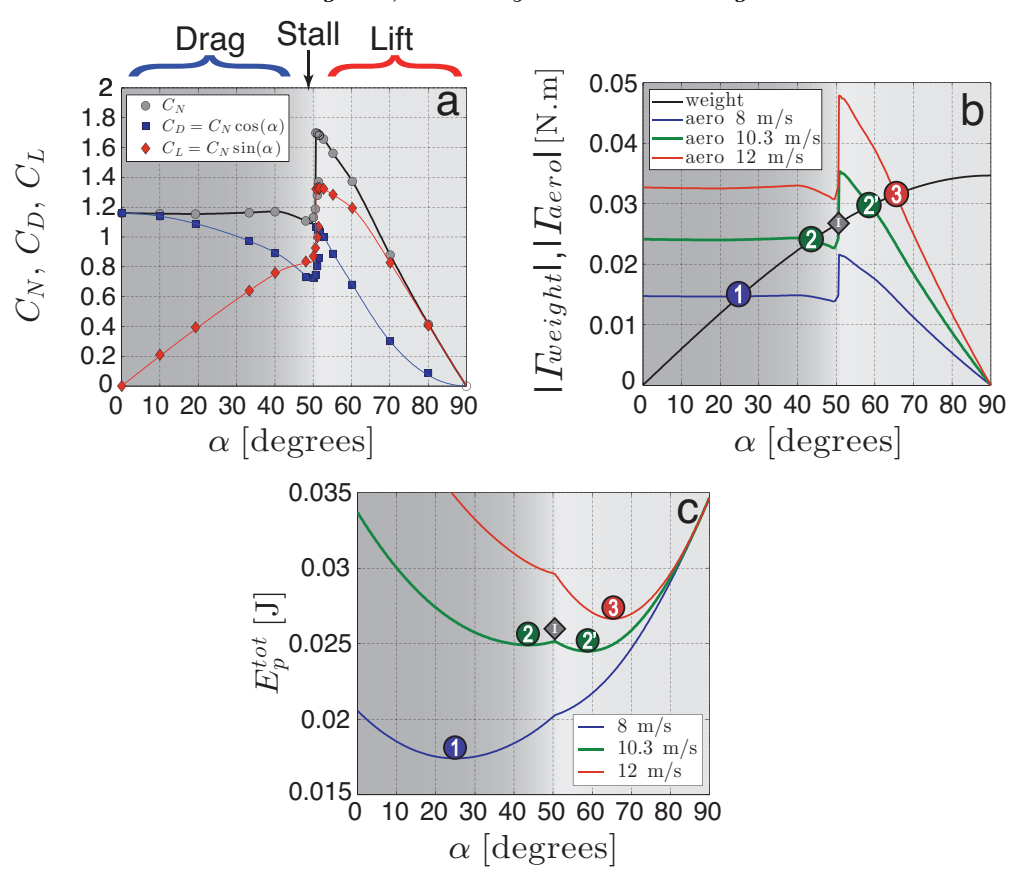

FiguRE 3. (a) Reference static measurements of normal drag coefficient $C_{N}$ for an inclined static circular cylinder as a function of inclination angle (gray circles) ; data is taken from Flachsbart (1932). Blue squares and red diamonds show the corresponding drag $\left(C_{D}\right)$ and lift $\left(C_{L}\right)$ coefficients. (b) Modulus of the moment of of gravity and of aerodynamic forces on the pendulum for three different wind velocities as a function of the inclination angle of the pendulum. Intersection points indicate equilibrium positions. (c) Total potential energy of the pendulum as a function of inclination angle.

branch occurs for a lower critical velocity $U_{\downarrow}^{\text {lam }} \simeq 10 \mathrm{~m} / \mathrm{s}$, where the equilibrium angle suddenly drops from around $60^{\circ}$ to around $45^{\circ}$. As a result, the pendulum is found to be bi-stable for wind velocities in the range $10-11.2 \mathrm{~m} / \mathrm{s}$, with an equilibrium position which depends on the history path of the system. We also observe a narrow band of forbidden angles which are never explored by the system.

\subsection{Origin of the bi-stability}

We will show that this peculiar behavior can be understood in aerodynamic terms, from the angular dependency of the normal drag coefficient $C_{N}$ of an inclined disk. $C_{N}$ is defined such that the aerodynamic force acting on the plate is $\vec{F}_{\text {aero }}=\frac{1}{2} \rho_{a i r} C_{N} S_{D} U^{2} \vec{e}_{N}$, with $\rho_{\text {air }}$ the density of air, $S_{D}=\pi D^{2} / 4$ the area of the disk and $\vec{e}_{N}$ the normal vector on the downstream face of the plate (see inset in figure 1). Figure 3a shows reference measurements of $C_{N}$ by Flachsbart (1932) (note that in the present study as in Flachsbart (1932) Reynolds number based on disk diameter $R_{D}=U D / \nu$ exceeds $10^{4}$, hence aerodynamic coefficients are assumed not to depend on $R e_{D}$ ). Figure 3 a shows that the angular dependency of $C_{N}$ exhibits a sharp discontinuity at a critical angle $\alpha^{\text {stall }} \simeq 51^{\circ}$. This corresponds to the stall transition of the plate: when the normal drag coefficient is split into the drag $\left(C_{D}=C_{N} \cos (\alpha)\right)$ and lift coefficients $\left(C_{L}=C_{N} \sin (\alpha)\right)$, as shown in figure $3 \mathrm{a}$, it becomes clear that for large inclinations $\alpha>\alpha_{\text {stall }}$ (i.e. for low angles of attack), lift dominates drag and vice versa. Lift is maximal for $\alpha \gtrsim \alpha^{\text {stall, }}$, where the disk is somehow in a flying configuration. $C_{L}$ abruptly drops when $\alpha$ decreases below $\alpha^{\text {stall }}$ (until it vanishes when the plate is perpendicular to the flow at $\alpha=0^{\circ}$ ). For 
low inclinations (i.e. large angles of attack) drag dominates lift. In the sequel we shall therefore speak of $d r a g$ dominated states when $\alpha<\alpha^{\text {stall }}$ and of lift dominated states when $\alpha>\alpha^{\text {stall }}$. Such a stall transition has been extensively studied in aeronautics as it is a major concern for airfoil design (Hoerner (1965)). Its physical origin, which goes beyond the scope of this article, is a complex (though relatively well understood) fluid mechanics problem related to massive flow separation at the backside of the plate (or airfoil). We point however that, contrary to the case of certain aeronautic airfoils (see for instance Mueller (1985); Mittal \& Saxena (2002)), the stall transition for a flat circular plate does not exhibit any noticeable hysteresis when the inclination angle is cycled up and down at constant velocity. The hysteretical behavior observed here for the pendulum is therefore not related only to aerodynamics. It actually relies on the additional angular degree of freedom of the pendular system, whose equilibrium angle $\alpha_{e q}$ self adjusts to ensure the balance between the moment of aerodynamic force $\left(\vec{\Gamma}_{\text {areo }}\right)$ and that of gravity $\left(\vec{\Gamma}_{\text {weight }}\right)$ :

$$
\begin{aligned}
\vec{\Gamma}_{\text {areo }} & =\frac{1}{2} \rho_{a i r} S_{d} C_{N}(\alpha) U^{2} \overline{O D} \cos \alpha \vec{e}_{x}, \\
\vec{\Gamma}_{\text {weight }} & =-m g \overline{O G} \sin \alpha \vec{e}_{x} .
\end{aligned}
$$

Figure $3 \mathrm{~b}$ shows the modulus of the aerodynamic moment acting on the pendulum for three different wind velocities $U$ and that of gravity (which is independent of flow velocity), as a function of inclination $\alpha$. The quantitative estimation of the aerodynamic moment requires to know the actual angular dependency of the normal drag coefficient $C_{N}(\alpha)$ : the reference measurements from Flachsbart (1932) (shown in 3a) have been used for this purpose. Depending on the flow velocity, gravity and aerodynamic curves can have one or multiple intersection points meeting the equilibrium condition $\left|\Gamma_{\text {aero }}\right|=\left|\Gamma_{\text {weight }}\right|$. For low velocities, they intersect only once for $\alpha_{e q}^{1}<\alpha_{\text {stall }}$ (point labeled " 1 " in the figure) and the equilibrium results mainly from a drag vs gravity balance. For large velocities, the intersection occurs for $\alpha_{e q}^{3}>\alpha_{\text {stall }}$ (point labeled " 3 ") and we have essentially a lift vs gravity equilibrium. For intermediate velocities, three intersection points exist: one at $\alpha_{e q}^{2} \lesssim \alpha_{\text {stall }}$ (labeled " 2 "), one at $\alpha_{e q}^{2^{\prime}} \gtrsim \alpha_{\text {stall }}$ (labeled " 2 '") and one at $\alpha_{I} \simeq \alpha_{\text {stall }}$ (labeled "I"). This situation corresponds to the range of bi-stability reported in figure 3a, where points " 2 " and " 2 " " are two stable states (respectively on the $d r a g$ and lift branches) and point "I " corresponds to an unstable equilibrium position of the pendulum close to the stall angle. This bi-stability scenario can be rewritten in terms of a two potential wells system, based on the total potential energy of the pendulum $E_{p}^{\text {tot }}(\alpha)=E_{p}^{\text {weight }}(\alpha)+E_{p}^{a e r o}(\alpha)$, where $E_{p}^{\text {weight }}(\alpha)$ is the gravity potential energy and $E_{p}^{a e r o}(\alpha)$ is the potential energy associated to aerodynamic force.

$$
\begin{aligned}
E_{p}^{\text {weight }}(\alpha) & =m g \overline{O G} \sin \alpha, \\
E_{p}^{\text {aero }}(\alpha) & =-\frac{1}{2} \rho_{a i r} S_{d} U^{2} \overline{O D} \int_{\alpha}^{\pi / 2} C_{N}\left(\alpha^{\prime}\right) \mathrm{d} \alpha^{\prime} .
\end{aligned}
$$

Note that aerodynamic potential energy $E_{p}^{\text {aero }}(\alpha)$ is defined so to be zero when the pendulum is aligned with the mean stream (i.e. $\alpha=\pi / 2$ ) which would be the natural equilibrium position in absence of gravity. Figure $3 \mathrm{c}$ shows the total potential energy $E_{p}^{t o t}(\alpha)$, as a function of inclination $\alpha$, for the same three velocities for which balance of moments has been analyzed in figure $3 \mathrm{~b}$. It shows that for the lower velocity only one potential well defines the stable drag dominated angular position of the system and that for the higher velocity only one potential well defines the stable lift dominated angular position. For the intermediate velocity two stable potential wells co-exist with an 
unstable equilibrium (local maximum of $E_{p}^{t o t}$ ) in between. For a more complete analysis, we show in figure 2 the iso-contour lines of the total potential energy in the $\alpha-U$ parameter space. Experimental measurements of equilibrium angle are found to lie almost perfectly on the path of local potential minima (emphasized by the solid line). The range of bi-stability is also well predicted by the two potential wells description, although the predicted hysteretical cycle is broader than what is experimentally observed. In particular the experimental transition from the lift branch down to the drag branch (when velocity is decreased below $U_{\downarrow}^{\text {lam }}$ ) occurs sooner in the experiment compared to the theory. This sooner transition results in an intrinsic narrow band of forbidden angles between approximately $51^{\circ}$ and $57^{\circ}$, which are never explored by the pendulum in the experiment. The reason of the anticipated lift to drag transition remains unexplained. First analysis of the pendulum fluctuations (see figure $4 \mathrm{~b}$ ), based on measurements of the standard deviation of the angular position of the pendulum, $\sigma_{\alpha}$, indicates that fluctuations in the laminar case are small $\left(\sigma_{\alpha}<1\right.$ degree in general and $\sigma_{\alpha} \simeq 0.1$ degree in the lift branch). It is therefore unlikely that fluctuations do activate here the anticipated departure from the lift branch. A possible explanation may be related to finite thickness effects (the thickness-over-diameter ratio of our plate is of the order of $4 \cdot 10^{-2}$ ) which may not be completely negligible when the edge of the plate faces the flow (as it is for the lifted case) and which may affect the stall transition compared to the reference measurements by Flachsbart (1932) who considered a plate with aspect ratio below $10^{-2}$. Recent numerical studies by Auguste et al. (2013) have shown for instance that such finite thickness effects still affect the free falling dynamics of flat disks with thickness-over-diameter ratios of the order of $10^{-1}$. Further experiments with different plate thicknesses will be carried to explore such possible effects on the pendular system.

\subsection{Influence of turbulence intensity}

We consider now the influence of enhanced free stream turbulence on the pendulum equilibrium. Figure 4a shows that a few percent of turbulence level (achieved with the passive grid) already has a measurable effect : while the drag branch remains almost unchanged compared to the non turbulent case, the lift branch is slightly decreased to lower equilibrium angles, and the bi-stability range is significantly reduced. Figure 4c shows the corresponding angular dependency for the normal drag coefficient $C_{N}(\alpha)$, inferred from the measurements of $\alpha_{e q}(U)$ by writing the equilibrium condition $\vec{\Gamma}_{a e r o}=$ $\vec{\Gamma}_{\text {weight }}$ (from eq. (3.1) and eq (3.2)) parametrized by $U$ :

$$
\left\{\begin{array}{l}
\alpha=\alpha_{e q}(U), \\
C_{N}(\alpha)=\frac{2 m g}{\rho_{a i r} S_{d} U^{2}} \frac{\overline{O G}}{O D} \tan \alpha,
\end{array}\right.
$$

(the solid line in figure $4 \mathrm{c}$ recalls the reference measurements of $C_{N}(\alpha)$ by Flachsbart (1932) previously discussed for the case of a laminar incident flow). At small inclinations $\left(\alpha<\alpha^{\text {stall }}\right)$, a few percent of turbulence marginally affects the drag coefficient, consistently with previous results for plates normal to the flow (see for instance Hoerner (1965)). At large inclinations on the contrary $\left(\alpha>\alpha^{\text {stall }}\right)$ aerodynamic lift is reduced up to $8 \%$ compared to the laminar case. Regarding the hysteresis and bi-stability reduction, we find it to occur through an anticipated drag to lift branch transition compared to the laminar case $\left(U_{\uparrow}^{\text {turb }}<U_{\uparrow}^{\text {lam }}\right)$, while the decreasing transition is unaffected by turbulence $\left(U_{\downarrow}^{\text {turb }} \simeq U_{\downarrow}^{\text {lam }}\right)$. The anticipation of the drag to lift transition is very likely activated by the enhanced turbulent fluctuations of the pendulum. This should be discussed together with figure $4 \mathrm{~b}$ and with the green curve in figure $3 \mathrm{c}$ which represents the laminar two potential wells landscape near $U_{\uparrow}^{\text {turb }} \simeq 10.3 \mathrm{~m} / \mathrm{s}$. We assume as a first approximation 

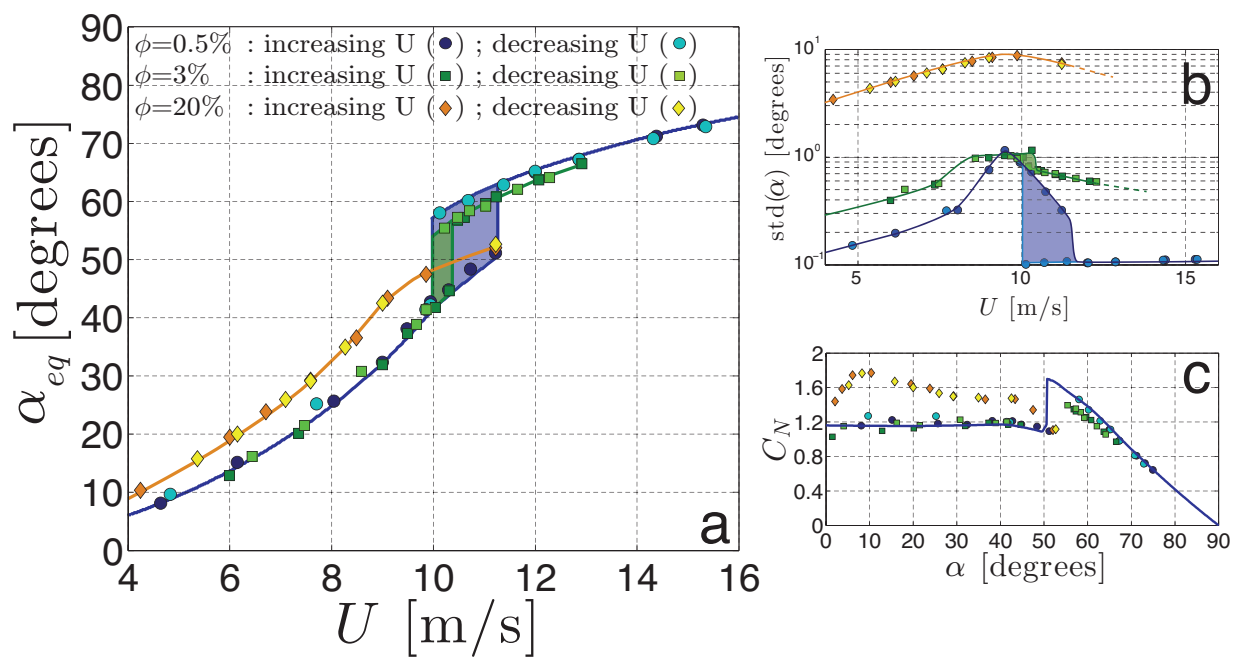

Figure 4. (a) Average angular equilibrium position of the pendulum as wind velocity in increased and decreased for the three different levels of turbulence $(\phi)$ explored. Blue and green coloured surfaces emphasize the hysteretic cycle for the laminar and moderate turbulence cases respectively. (b) Corresponding standard deviation of the fluctuations of the angular position of the pendulum. (c) Angular dependency of normal drag coefficient of the plate estimated from the angular average position of the pendulum $\alpha_{e q}$. The blue solid line recalls the laminar static measurements of $C_{N}$ by Flachsbart (1932) already shown in figure 3a.

that turbulence acts as an additive noise on this unmodified potential landscape (although strictly speaking, we know that the potential energy in the lift branch is slightly modified due to the reduction of the lift coefficient previously mentioned). It can be seen in figure $3 \mathrm{c}$ that the drag potential well is very shallow at $10.3 \mathrm{~m} / \mathrm{s}$, hence the escape from this well can be easily activated by enhanced fluctuations. Besides, the inspection of the standard deviation of the pendulum angular position (figure $4 \mathrm{~b}$ ) in the moderate turbulence case shows that at this same wind velocity, $\sigma_{\alpha}$ is about $50 \%$ larger than in the laminar case. Regarding the lift to drag transition, figure $4 \mathrm{~b}$ shows that the fluctuation level in the turbulent lift branch is comparable to that of the laminar drag branch in the vicinity of the turbulent hysteretical cycle. Considering that, as shown in figure 3c, the lift potential well is deeper than the $d r a g$ potential well at $U_{\uparrow}^{t u r b} \simeq 10.3 \mathrm{~m} / \mathrm{s}$ (this asymmetry has been checked to prevail even if the potential energy is estimated using the reduced lift coefficient), and that no escape from the drag potential well has been observed in the laminar case at this wind speed, it is reasonable that for a similar level of fluctuations the pendulum does not escape from the lift potential and hence $U_{\downarrow}^{t u r b}<U_{\uparrow}^{t u r b}$. It is however important to stress at this point that a more complete understanding of the role of fluctuations on the hysteretical behavior requires a more detailed statistical analysis, taking in consideration not only the standard deviation of fluctuations, but also higher moments, as the skewness (which characterize the asymmetry of the fluctuations) and the flatness (to investigate the possible occurrence of strong intermittent events) which may play a crucial role in transition processes between the two potential wells. Such a detailed investigation will be addressed in forthcoming studies.

When the turbulence level is further increased up to $\phi=20 \%$ (with the active grid) the scenario drastically changes. No clear transition between a drag branch and a lift branch is visible anymore and, bi-stability and hysteresis have disappeared. Due to mechanical power limitations, the wind speed could not be pushed above $11 \mathrm{~m} / \mathrm{s}$ with the active grid 
operating. Therefore we cannot be conclusive regarding a possible transition toward a lift branch at higher wind velocities; however this seems unlikely, considering that at $11 \mathrm{~m} / \mathrm{s}$ the transition was already observed for $\phi=3 \%$. The high turbulence case also exhibits a significant increase of the equilibrium angle of the drag branch compared to lower turbulence cases. This corresponds to a significant increase of normal drag coefficient $C_{N}$, up to $50 \%$ as shown in the inset of figure 4 . As for the moderate turbulence case, it is enlightening to consider the fluctuations of the pendulum, reported in figure $4 \mathrm{~b}$. It can be seen that the standard deviation of these fluctuations is now one order of magnitude larger. It attains values as large as 10 degrees. The amplitude of the fluctuations is therefore comparable to the angular extent of the hysteresis cycle reported in the laminar case, which is therefore completely smeared out. Further experiments are planned, where much longer time series will be recorded, in order to improve the statistical description of the pendulum fluctuations. An important aspect to be addressed concerns the question if the laminar bi-stability and hysteresis may remain reminiscent as a broad a bi-modal distribution of the pendulum fluctuations or if on the contrary the dramatic change of the turbulent drag coefficient has killed all reminiscence of the laminar features.

\subsection{Discussion}

Our results are worth being briefly discussed in the context both of fundamental aspects of bi-stable systems and of practical implications for hydro and aerodynamic issues. Concerning the first aspect, our system offers a simple experimental model for the investigation of fundamental properties of bi-stable and hysteretical processes. In particular, it has the capability to simply address questions related to stochastic processes in bi-stable systems, what remains an active field of research and an archetype model for many problems in physics, chemistry, geophysics and biology (Van Kampen (2007)). In the present work for instance turbulence plays the role of noise. In the moderate turbulence case $(\phi=3 \%)$, velocity fluctuations mostly act as an additive noise on the aerodynamic force acting on the disk $\left(F_{\text {aero }}=1 / 2 \rho C_{N} S_{d} U^{2}\right)$, as small velocity fluctuations can be linearized with respect to the perturbation $\phi<<1$. Turbulence can then be regarded as a heat bath where the reduction of hysteresis reported in figure 4 illustrates the thermal activation (Kramers (1940); Van Kampen (2007)) induced by additive noise which increases the escape probability from one potential well to the other. The case of high turbulence level is more complex and cannot be simply linearized as an additive noise. Multiplicative effects (resulting in fluctuations of the energy barrier itself between potential wells) are very likely involved, and require further investigations.

From the aero/hydro-dynamic point of view, learnings from this simple experiment are also rich. As already discussed from the inset of figure 4, the angle of equilibrium of the pendulum gives a direct measurement of the normal drag and hence of the lift and drag coefficients of the inclined plate. The impact of turbulence on such coefficients in general remains an open question with direct energetic and structural implications in aeronautics, civil engineering and turbulent transport phenomena. While evidence of lift reduction by turbulence is well documented in airfoil research literature, the impact of turbulence on drag remains debated. Numerical studies on the drag coefficient of a sphere are for instance controversial as Bagchi \& Balachandar (2003) report that mean drag is not affected by turbulence intensity, while more recent studies by Homann et al. (2013) report a significant enhancement of drag with increasing turbulence intensity. Though the quantitative values of lift reduction and drag enhancement by turbulence reported in the present study may change for other geometries than an inclined disk, our measurements reveal the necessity of a careful consideration of turbulent effects for instance in architectural design, spoiler conception and vehicle aerodynamic optimization. 
Besides, our results directly concern fluid-structure interaction (FSI) properties of flat pendular systems, which are common in many applications, including spoilers, industrial check valves and artificial heart valves. The fact that their equilibrium is bi-stable have important practical implications. For instance, pendular plate anemometers, as imagined by Alberti, da Vinci and Hooke, become intrinsically ineffective as in the bi-stable range, the angle of the pendulum is not univocally related to flow velocity. But beyond this, incorporating the bi-stability mechanism in FSI analysis may significantly improve the understanding and predictability of such systems. This is particularly important for the design of tilting disk and swing check valves, which are crucial elements of nuclear plant security systems (Lim et al. (2006); McElhaney (2000)), but also of prosthetic heart valves (Guivier-Curien et al. (2009); Sotiropoulos \& Borazjani (2009)). Bi-stability implies an intrinsic limitation of static FSI models, and the requirement of a dynamic modeling, to properly account for the history of the system, as the flow rate in the valve cycles up and down. With this respect, the pendular system investigated here, also gives a simple and quantitative benchmark to test the accuracy of FSI models. Alternatively, the two-well potential approach gives a possible strategy to replace complex and computationally expensive dynamical FSI simulations by an empirical model based on static macroscopic data of the angular dependency of hydro/aerodynamic coefficients, which may be relevant to investigate valve dynamics and stability. Finally our results also show how design strategies may include possible effects of fluctuations which can help reducing hysteresis or improving the opening of the valve at low flow rates as a result of turbulent drag enhancement.

\section{Conclusion}

To summarize, we have evidenced the bi-stability and hysteresis of a flat pendulum facing a flow. We have given a quantitative interpretation of the phenomenon (for the case of a laminar incident flow) where the complex hydrodynamical interaction of the plate with the fluid is reduced to a simple two potential wells model mainly defined by the plate normal drag coefficient. The academic attractiveness of the pendulum paradigm makes this configuration ideal for pedagogical purposes as well as for fundamental researches on stochastic processes in bi-stable systems. Present work has focused only on the quasi-steady equilibrium of the pendulum, but much remains to be done regarding to dynamical features and fluctuations. We will for instance carry further experiments (with very long temporal recordings), to monitor the dynamics of the noisy pendulum in the vicinity of the bi-stable range, in order to better quantify the actual impact of fluctuations on the transition between branches and to extract escape time statistics. This question, known as Kramers problem (Kramers (1940)), remains a classical problem of stochastic multi-stable systems. An interesting point to be addressed in our particular configuration concerns the influence of specific properties of the forcing noise. In the laminar case resonant effects between periodic structures shedded downstream the plate and natural oscillations of the pendulum, may for instance be important. In the turbulent case, we expect transitions between the two stable states to be also affected by timecorrelation properties of turbulence (which define the color of the imposed noise, but also by spatial-correlation effects (related for instance to the ratio between the integral scale of the surrounding turbulence and the size of the pendulum) as well as by intermittency effects. Beyond the present configuration where additive noise is introduced by moderate turbulence, the versatility of our system allows to investigate the influence of several parameters, such as amplitude and color of noise (which can be simply introduced by a controlled random mechanical forcing of the pendulum), but also of the shape of 
the potential well itself (which can be tailored by adding a torsional spring or a magnetic force for instance). The ability of the bi-stable pendulum to undergo stochastic resonance will also be investigated.

\section{REFERENCES}

Auguste, Franck, Magnaudet, Jacques \& Fabre, David 2013 Falling styles of disks. Journal of Fluid Mechanics 719, 388-405.

BAGChi, P \& Balachandar, S 2003 Effect of turbulence on the drag and lift of a particle. Physics of Fluids 15 (11), 3496-3513.

Bolster, Diogo, Hershberger, Robert E. \& Donnelly, Russell J. 2010 Oscillating pendulum decay by emission of vortex rings 1. Physical Review E 81, 1-6.

Chrust, Marcin, Bouchet, Gilles \& Dusek, Jan 2013 Numerical simulation of the dynamics of freely falling discs. Physics of Fluids 25 (4), 044102.

Flachsbart, Von O. 1932 Messungen an ebenen und gewölbten Platten. In Ergenbisse der Aerodynamischen Versuchsanstalt zu Göttingen, IV., Ava edn. (ed. L. Prandtl), pp. 96100. Gottingen: Verlag von R. Oldenburg.

Guivier-Curien, Carine, Deplano, Valérie \& Bertrand, Eric 2009 Validation of a numerical 3-D fluid-structure interaction model for a prosthetic valve based on experimental PIV measurements. Medical engineering $\& 3$ physics 31 (8), 986-993.

Hoerner, S. F. 1965 Fluid-dynamic drag: practical information on aerodynamic drag and hydrodynamic resistance. Published by the author.

Homann, Holger, Bec, JÉrémie \& Grauer, Rainer 2013 Effect of turbulent fluctuations on the drag and lift forces on a towed sphere and its boundary layer. Journal of Fluid Mechanics 721, 155-179.

Kramers, H.A. 1940 Brownian motion in a field of force and the diffusion model of chemical reactions. Physica 7 (4), 284-304.

Lim, Ho-Gon, Park, Jin-Hee \& JAng, Seung-Cheol 2006 Development of a swing check valve model for a low velocity pipe flow prediction. Nuclear Engineering and Design 236 (10), $1051-1060$

MCELhaney, K.L 2000 An analysis of check valve performance characteristics based on valve design. Nuclear Engineering and Design 197 (1-2), 169-182.

Mittal, S. \& Saxena, P. 2002 Prediction of hysteresis associated with the static stall of an airfoil. AIAA Journal 38, 933-935.

Mueller, T. J. 1985 The influence of laminar separation and transition on low Reynolds number airfoil hysteresis. Journal of aircraft 22 (9), 763-770.

Neill, Douglas, Livelybrooks, Dean \& Donnelly, Russell J 2007 A pendulum experiment on added mass and the principle of equivalence. American Journal of Physics 75 (March), 226-229.

Obligado, Martin, Missaoui, Mahrane, Monchaux, Romain, Cartellier, Alain \& Bourgoin, MickAËL 2011 Reynolds number influence on preferential concentration of heavy particles in turbulent flows. Journal of Physics: Conference Series 318 (5), 052015.

Sotiropoulos, Fotis \& BorazJAni, Iman 2009 A review of state-of-the-art numerical methods for simulating flow through mechanical heart valves. Medical $\& 6$ biological engineering $\&$ computing 47 (3), 245-56.

Van Kampen, N. G. 2007 Stochastic Processes in Physics and Chemistry. Elsevier. 